\title{
Capturing the Cacophony of Movement
}

Citation for published version (APA):

Mentzel, T. (2018). Capturing the Cacophony of Movement: Assessment, epidemiology and treatment of movement disorders in patients with psychotic disorders. [Doctoral Thesis, Maastricht University]. Datawyse / Universitaire Pers Maastricht. https://doi.org/10.26481/dis.201800607tm

Document status and date:

Published: 01/01/2018

DOI:

10.26481/dis.201800607tm

Document Version:

Publisher's PDF, also known as Version of record

\section{Please check the document version of this publication:}

- A submitted manuscript is the version of the article upon submission and before peer-review. There can be important differences between the submitted version and the official published version of record.

People interested in the research are advised to contact the author for the final version of the publication, or visit the DOI to the publisher's website.

- The final author version and the galley proof are versions of the publication after peer review.

- The final published version features the final layout of the paper including the volume, issue and page numbers.

Link to publication

\footnotetext{
General rights rights.

- You may freely distribute the URL identifying the publication in the public portal. please follow below link for the End User Agreement:

www.umlib.nl/taverne-license

Take down policy

If you believe that this document breaches copyright please contact us at:

repository@maastrichtuniversity.nl

providing details and we will investigate your claim.
}

Copyright and moral rights for the publications made accessible in the public portal are retained by the authors and/or other copyright owners and it is a condition of accessing publications that users recognise and abide by the legal requirements associated with these

- Users may download and print one copy of any publication from the public portal for the purpose of private study or research.

- You may not further distribute the material or use it for any profit-making activity or commercial gain

If the publication is distributed under the terms of Article $25 \mathrm{fa}$ of the Dutch Copyright Act, indicated by the "Taverne" license above, 
Summary and general discussion 

Since the discovery of chlorpromazine in 1950, antipsychotics play a crucial role in the treatment of psychotic disorders[1]. Since then many new antipsychotics have been introduced to the market. They are commonly categorized into First-Generation Antipsychotics (FGAs) and Second-Generation Antipsychotics (SGAs). FGAs were developed in the 1950s and 1960s and the SGAs emerged in the 1980s[1].

Although FGA are very effective in the treatment of psychotic disorders, many patients developed movement disorders as a side effect[2]. However, an exception, already developed in the sixties, was clozapine, a new antipsychotic that induced far less movement disorders than FGAs. Clozapine was considered as the first of a new generation of antipsychotics[3, 4].

The most common movement disorders observed in patients with psychotic disorders are parkinsonism, akathisia, dyskinesia and dystonia[2, 5]. These movement disorders are a serious burden on patients; they can cause distress (especially feelings of shame) and (physical) discomfort in patients and often contribute to medication noncompliance[5].

This chapter begins with a summary of our studies on the epidemiology of movement disorders in the GROUP study and the switch to clozapine monotherapy as a treatment for tardive dyskinesia. The second part of this chapter summarizes our research on the use of digital/electronic instruments to assess and monitor movement disorders.

\section{Epidemiology of Movement Disorders}

The epidemiology of movement disorders in patients on long-term treatment with first generation antipsychotics has been studied thoroughly[2,6]. However, less is known about the incidence and prevalence of movement disorders in patients who just started treatment with antipsychotics, and especially with second-generation antipsychotics[7]. The first years of treatment are especially interesting to study, as the neural pathways that are associated with movement disorders are still relatively unaffected by the longterm potentiation effects of antipsychotic treatment. Therefore, we studied the incidence, prevalence, persistence and clinical correlates of parkinsonism, akathisia, dyskinesia and dystonia in a large cohort of relatively young adults, the Genetic Risk and Outcome of Psychosis (GROUP) study[8]. Surprisingly, we found that although the majority of patients in the cohort were treated exclusively with SGAs as many as $40 \%$ developed a movement disorder. The incidence, prevalence and persistence rates of movement disorders in the GROUP study are lower than the rates reported by studies that investigated older populations treated with first generation antipsychotics[2, 5, 6, $9,10]$ and slightly higher than studies investigating SGAs in similar populations[4, 11]. The severity of parkinsonism was correlated to IQ scores, severity of akathisia, severity of dyskinesia, and the severity of the negative symptoms of schizophrenia (i.e. reduc- 
tions in emotional responsiveness, motivation and both verbal and physical expression). This is in line with other studies [6, 12-15]. Correlations were also observed between the degree of the positive symptoms of schizophrenia (i.e. delusions and hallucinations) and the severity of akathisia and dyskinesia. Another interesting finding in our study was the absence of a dose-response relation between antipsychotics and movement disorders. The association between the severity of movement disorders and the type of antipsychotic medication was also absent. Yet, we did observe that the patients treated with more than one antipsychotic drug had more severe movement disorders than the patients treated with a single antipsychotic. Altogether, these findings show that movement disorders are frequently present in a large, relatively young population that predominantly used SGAs. Thus movement disorders remain a serious concern as they negatively impact the quality of life of patients. This underscores the importance of monitoring for movement disorders in patients with schizophrenia or other psychotic disorders.

\section{The effect of switching to clozapine treatment on Tardive Dyskinesia}

Tardive dyskinesia develops months to years after antipsychotic treatment has been started and it can persist after discontinuation of treatment $[5,10]$. Tardive dyskinesia is believed to be caused by a long-term effect of antipsychotic treatment on the parts of the dopamine system involved in regulating movement[16, 17]. Two dominant etiological hypotheses of tardive dyskinesia are a dysregulation of neurons in the basal ganglia involved with motor control either due to hyper-sensitization or cell damage caused by the production of free radicals[16, 17]. Several proposed treatments for tardive dyskinesia (i.e. anticholinergics, benzodiazepines, calcium channel blockers and vitamin $\mathrm{E}$ supplementation) lack convincing evidence[18-21]. Switching current antipsychotic treatment to clozapine monotherapy has also been suggested as a potential treatment for tardive dyskinesia[16, 22, 23]. However, there are no meta-analyses dedicated to investigating clozapine as a potential treatment for tardive dyskinesia. Therefore, we performed a meta-analysis to study the effect of switching to clozapine on the severity of tardive dyskinesia. We opted to use a broad search strategy that included studies that investigated the effect of switching to clozapine with tardive dyskinesia as the primary study outcome and studies with tardive dyskinesia as a secondary outcome. The reason we also included studies with tardive dyskinesia as a secondary outcome was because we expected to find few studies that investigated the switch to clozapine as a treatment for tardive dyskinesia and the findings of the studies with tardive dyskinesia as a secondary outcome could have contradicted the findings of the other studies. This search returned more than 10.000 hits of which only 17 studies met our inclusion criteria and could be included in our meta-analysis. Only 4 of the 17 studies investigated tardive dyskinesia as a primary outcome. These four studies showed, convincingly, that 
switching to clozapine can substantially reduce the severity of moderate to severe tardive dyskinesia, and in many cases even alleviate dyskinetic symptoms completely. The other studies showed that a switch to clozapine monotherapy is not likely to worsen the severity of tardive dyskinesia in patients with mild to no dyskinetic symptoms. Thus, our findings make a strong case for switching to clozapine monotherapy to treat tardive dyskinesia in patients with moderate to severe dyskinetic symptoms.

\section{Instrumental Assessments of Movement disorders}

When studying movement disorders it is important to quantify their severity using valid and reliable methods. The second part of this thesis focuses on using digital/electronic instruments to assess and monitor movement disorders. A wide range of digital/electronic instruments have already been developed to assess and monitor movement disorders. However, the adoption of these instruments into clinical practice has been minimal. Therefore, we set out to develop a number of instrumental assessments suitable for use in a regular clinical setting. We aimed to keep these instruments easy to use and affordable while being more sensitive and reliable than conventional scales. Four instruments were developed that utilize the latest technologies: (i) A bradykinesia assessment that is similar to conventional observer based scales, but uses inertial sensors to assess the severity of bradykinesia. (ii) A redesign of an instrument that measures variability in force, a sign of dyskinesia. (iii) An instrument that uses a depth camera to automate the assessment of orofacial dyskinetic signs. (iv) An app that includes several touch based tasks aimed at assessing signs of movement disorders and other soft motor signs (Neurological Soft Signs). Four studies were conducted with these instruments, three studies investigated the feasibility of these instruments (i, iii \& iv) and one study determined the validity and reliability of the instrument for assessing bradykinesia (i).

Recent studies have shown that inertial sensors can reliably capture and assess the movements of tasks used by conventional rating scales that assess the motor signs of Parkinson's Disease[24-26]. Considering that the signs of parkinsonism are very similar to those of Parkinson's Disease we investigated the feasibility of an instrument for the assessment of antipsychotic-induced bradykinesia. Performances on a wide range of motor tasks were compared between six bradykinetic patients and five healthy controls. These tasks were derived from common observation based rating scales such as the St. Hans Rating Scale and the Unified Parkinson's Disease Rating Scale. The patients performed significantly worse than the controls on seven of the thirteen tasks. The combination of tasks we deem the most feasible includes walking 20 metres, rapidly bending/stretching the elbow, rotating the lower arm and stomping the foot on the ground. Combining these four tasks distinguished patients from controls very effectively. The validity and reliability of these four tasks were determined in a subsequent study. To 
investigate the validity of the bradykinesia assessment by our instrument, we determined its concurrent validity with the bradykinesia items of the Unified Parkinson's Disease Rating Scale in 64 in-patients with a psychotic disorder and varying degrees of bradykinesia. Our findings show that the validity of the instrument's assessments is high. We also found that the instrument's test-retest reliability is excellent. This shows that it is feasible to assess antipsychotic-induced bradykinesia validly and reliably using our instrument.

Reliable assessment of the orofacial signs of dyskinesia is difficult and requires a substantial frame of reference. Inspired by a previous study we designed a method to automate the assessment of dyskinetic movements that occur in the facial musculature[27]. Instead of using a regular camera we used an infrared depth camera, as this type of camera can reliably capture facial movement without the need to place markers. The faces of five dyskinetic patients and five healthy controls were filmed with a Kinect camera while they rested or performed a motor task to distract them. The data recorded by the Kinect showed that there was significantly more movement in the lips of the patients than of the controls. In other regions of the face differences were not significant, because the Kinect could not detect subtle movements reliably enough in these regions. Currently, it is not yet feasible to assess orofacial dyskinesia, as not all signs can be recorded reliably. However, it is likely that this method will soon become more feasible with improvements to the algorithm that tracks the movement of the facial regions and increases in the resolution of depth cameras.

The widespread adoption of smartphones and tablets make them ideal for screening motor signs in large groups. Screening for motor symptoms could help improve the detection of individuals at risk of developing a psychotic disorder[28]. Several signs related to movement disorders and neurological soft signs are elevated in these individuals in comparison to their healthy peers[29, 30]. We developed an app that uses several touchscreen based tasks derived from existing instruments and rating scales. The feasibility of these tasks was determined by first investigating whether these tasks are capable of reliably detecting very subtle differences in these motor signs. To determine the discriminability and reliability of these tasks we compared scores on these tasks between 10 young and 6 middle-aged adults. The results demonstrate that several of these tasks are capable of detecting very subtle differences in reaction time, movement speed and the planning and accuracy of movement. As the reaction time, the tracing and the trail making tasks differed significantly between young and middle-aged adults. The discriminability, reliability, ease of use and low cost of this tablet based assessment warrants further research; further investigation whether or not these tasks can be used to screen for subtle motor signs in individuals with an increased risk of developing a psychotic disorder is recommended. 


\section{General Discussion}

This thesis expands the knowledge of movement disorders related to psychosis in epidemiological, treatment and diagnostic areas. First, a large prospective study in a relatively young population using predominantly SGA shows that movement disorders are frequently present. Second, a meta-analysis of the effect of a switch to clozapine in patients with tardive dyskinesia shows that this intervention is very effective. Third, innovative and feasible instruments have been developed to measure parkinsonism and tardive dyskinesia.

\section{GROUP study}

The epidemiological study of movement disorders in the GROUP cohort shows that, in contrast to what is often suggested, movement disorders are also frequent in young adults treated with SGAs. A surprising outcome of the investigation of the clinical correlates of movement disorders was that there was no significant association between the type of antipsychotic treatment, FGA or SGA, and the severity of movement disorders. An explanation for the lack of the association between antipsychotic treatment and severity could be that it was overseen due the combination of the relatively long time (three years) between measurements and the naturalistic design of the GROUP study. On the other hand, it may be possible that not all movement disorders are directly caused by antipsychotic treatment but that they are integral symptoms to schizophrenia. This idea is supported by antipsychotic naïve patients with a first episode psychotic disorder, who also had movement disorders [31]. Moreover, our results show that parkinsonism may be interrelated with the mental and cognitive symptoms of schizophrenia. Both findings support the hypothesis that movement disorders are, at least partially, integral to schizophrenia. Considering that nearly $40 \%$ of the patients in the GROUP cohort had a movement disorder, there is a strong need to regularly monitor movement disorders in patients with psychotic disorders. Even if the actual prevalence of movement disorders may be somewhat lower than our findings suggest $[4,11]$ they are still a frequent and serious burden.

\section{Tardive dyskinesia}

Tardive dyskinesia can be a very persistent movement disorder. Several studies have investigated treatments for managing its symptoms[18-21], but these treatments lack convincing evidence. The results from our meta-analysis show that switching to clozapine can effectively reduce dyskinetic symptoms. This is remarkable because with the exception of olanzapine[22] this does not appear to be the case for other antipsychot- 
ics. The mechanisms behind the effectivity of clozapine as a treatment for tardive dyskinesia remains unknown. A possible explanation is that clozapine's unique receptor profile or that its cholinergic effects revert the hypersensitisation or damage of the striatal neurons involved with movement. However, neither PET nor SPECT imaging studies clearly support these explanations[32]. Another explanation for the efficacy of clozapine, yet to be explored by PET studies, is that clozapine may regulate presynaptic dopaminergic function differently than other antipsychotics[32]. Clozapine is a very effective antipsychotic, but a switch to clozapine has some drawbacks. Clozapine may induce or worsen metabolic problems and requires regular blood tests due to the increased risk of agranulocytosis[33]. Therefore, clozapine is typically only prescribed when patients do not respond to, or do not tolerate other antipsychotics. However, there are many cases in which the burden of TD outweighs the risks of clozapine treatment and in these cases switching to clozapine could provide considerable relief.

\section{Instrumental assessment of movement disorders}

There are some practical inconveniences to regular monitoring of movement disorders. Monitoring can be time consuming and expensive, as it requires regular training of staff to achieve valid and reliable results. Fortunately, this can be improved substantially with the application of instrumental assessments. These instruments can make screening of movement disorders easier and more efficient and the reliability of these assessments is far less dependent on the experience and training of the assessor. Thus, monitoring can be off loaded to other staff members, freeing up the costly time of clinicians. There are even instruments that are capable of registering signs of movement abnormalities that are too subtle to be detected by the clinical rating scales. The potential of these instruments is tremendous as more and more studies show that movement disorders are more than just a side effect of the antipsychotic medication[31, 34-36]. For druginduced movement disorders detecting subtle signs can help to detect and prevent movement disorders from developing further by treating them earlier.

Subtle movement disorders that are present in antipsychotic naïve patients of patients at risk for psychosis have prognostic value[37]. In patients at risk of psychosis subtle movement disorders predict conversion to psychosis and in antipsychotic naïve patients, parkinsonian symptoms predict worse outcome compared to antipsychotic naïve patients without parkinsonism[38-42]. The association between movement disorders and the severity of psychosis has also been shown in brain imaging studies, the presence of subtle movement disorders in the early stages (pre-clinical) of psychotic disorders has been been linked to structural brain abnormalities related to the severity of positive and negative symptoms[35].

Another advantage of detecting movement disorders is that they are objective signs in contrast to most other symptoms in individuals with pre-clinical psychotic disorders 
that are obtained with questionnaires. Screening for movement disorders in these groups may improve the specificity of a screening process. Nevertheless, clinical adoption of instrumental assessments for movement disorders remains limited. Instruments intended for routine assessment of movement disorders in a clinical setting must register movement disorders reliably, while they also must be affordable, portable and user friendly. Not all instruments meet these criteria, but with the introduction of novel instrumental assessments this is changing rapidly.

We predict that the implementation of devices and instruments will become widespread in clinical practice over the next decade. Our expectations are that using the next iterations of wearable devices such as smartwatches, fitness trackers and smart textiles in combination with machine learning algorithms will greatly improve the assessment and monitoring of movement disorders. The ability to assess movement disorders continuously can place them in context with the fluctuations of other symptoms (mental and cognitive), potential biomarkers (proteins in sweat[43]), and external influences.

This thesis has shown that movement disorders not only occur frequently in elderly patients on FGAs but also in younger patients on SGAs, that switching to clozapine is an important consideration for the treatment of tardive dyskinesia, and that instrumental assessment is an accurate and valid way for monitoring movement disorders. It would be interesting to see whether improvements in the instrumental assessment of movement disorders may change the outcome of patients with psychotic disorders. Would early detection prevent the development of drug induced movement disorders or the conversion to a psychotic disorder or improve the outcome of a first episode?

Another interesting direction for future research would be to gain more insights into the prognostic potential of monitoring and screening for movement disorders. Future studies could combine daily assessments of movement disorders with daily assessments of mental and cognitive symptoms, related biomarkers and changes in the environment via a combination of wearable sensors and experience sampling. This will lead to greater understanding of the course of schizophrenia and could establish movement disorders as an objective non-mental sign with high clinical relevance[42]. 


\section{References}

1. Shen, W.W., A history of antipsychotic drug development. Compr Psychiatry, 1999. 40(6): p. 407-14.

2. van Harten, P.N., et al., The prevalence of tardive dystonia, tardive dyskinesia, parkinsonism and akathisia The Curacao Extrapyramidal Syndromes Study: I. Schizophr Res, 1996. 19(2-3): p. 195-203.

3. Correll, C.U., S. Leucht, and J.M. Kane, Lower risk for tardive dyskinesia associated with secondgeneration antipsychotics: a systematic review of 1-year studies. Am J Psychiatry, 2004. 161(3): p. 41425.

4. Kahn, R.S., et al., Effectiveness of antipsychotic drugs in first-episode schizophrenia and schizophreniform disorder: an open randomised clinical trial. Lancet, 2008. 371(9618): p. 1085-97.

5. Chen, J.J. and D.M. Swope, Movement Disorders, in Drug-induced diseases : prevention, detection, and management, J.E. Tisdale and D.A. Miller, Editors. 2010, American Society of Health-System Pharmacists: Bethesda, Md. p. 211-235.

6. Novick, D., et al., Incidence of extrapyramidal symptoms and tardive dyskinesia in schizophrenia: thirtysix-month results from the European schizophrenia outpatient health outcomes study. J Clin Psychopharmacol, 2010. 30(5): p. 531-40.

7. Gebhardt, S., et al., Prevalence of movement disorders in adolescent patients with schizophrenia and in relationship to predominantly atypical antipsychotic treatment. Eur Child Adolesc Psychiatry, 2006. 15(7): p. 371-82.

8. Korver, N., et al., Genetic Risk and Outcome of Psychosis (GROUP), a multi-site longitudinal cohort study focused on gene-environment interaction: objectives, sample characteristics, recruitment and assessment methods. Int J Methods Psychiatr Res, 2012. 21(3): p. 205-21.

9. Bakker, P.R., et al., Predicting the incidence of antipsychotic-induced movement disorders in long-stay patients: A prospective study. Epidemiol Psychiatr Sci, 2013. 22(4): p. 375-9.

10. van Harten, P.N., et al., Incidence of tardive dyskinesia and tardive dystonia in African Caribbean patients on long-term antipsychotic treatment: the Curacao extrapyramidal syndromes study V. J Clin Psychiatry, 2006. 67(12): p. 1920-7.

11. Rybakowski, J.K., et al., Extrapyramidal symptoms during treatment of first schizophrenia episode: results from EUFEST. Eur Neuropsychopharmacol, 2014. 24(9): p. 1500-5.

12. Byne, W., et al., Prevalence and correlates of parkinsonism in an institutionalized population of geriatric patients with chronic schizophrenia. Int J Geriatr Psychiatry, 2000. 15(1): p. 7-13.

13. Miller, D.D., et al., Clinical correlates of tardive dyskinesia in schizophrenia: baseline data from the CATIE schizophrenia trial. Schizophr Res, 2005. 80(1): p. 33-43.

14. Tenback, D.E., P.N. van Harten, and J. van Os, Non-therapeutic risk factors for onset of tardive dyskinesia in schizophrenia: a meta-analysis. Mov Disord, 2009. 24(16): p. 2309-15.

15. Thanvi, B. and S. Treadwell, Drug induced parkinsonism: a common cause of parkinsonism in older people. Postgrad Med J, 2009. 85(1004): p. 322-6.

16. Hazari, N., N. Kate, and S. Grover, Clozapine and tardive movement disorders: a review. Asian J Psychiatr, 2013. 6(6): p. 439-51.

17. Louza, M.R. and D.P. Bassitt, Maintenance treatment of severe tardive dyskinesia with clozapine: 5 years' follow-up. J Clin Psychopharmacol, 2005. 25(2): p. 180-2.

18. Bhoopathi, P.S. and K. Soares-Weiser, Benzodiazepines for neuroleptic-induced tardive dyskinesia. Cochrane Database Syst Rev, 2006(3): p. CD000205.

19. Essali, A., et al., Calcium channel blockers for neuroleptic-induced tardive dyskinesia. Cochrane Database Syst Rev, 2011(11): p. CD000206.

20. Soares, K.V. and J.J. McGrath, Anticholinergic medication for neuroleptic-induced tardive dyskinesia. Cochrane Database Syst Rev, 2000(2): p. CD000204.

21. Soares-Weiser, K., N. Maayan, and J. McGrath, Vitamin E for neuroleptic-induced tardive dyskinesia. Cochrane Database Syst Rev, 2011(2): p. CD000209. 
22. Chakos, M., et al., Effectiveness of second-generation antipsychotics in patients with treatment-resistant schizophrenia: a review and meta-analysis of randomized trials. Am J Psychiatry, 2001. 158(4): p. 518-26.

23. Larach, V.W., et al., New strategies for old problems: tardive dyskinesia (TD). Review and report on severe TD cases treated with clozapine, with 12, 8 and 5 years of video follow-up. Schizophr Res, 1997. 28(2-3): p. 231-46.

24. Patel, S., et al., Monitoring Motor Fluctuations in Patients With Parkinson's Disease Using Wearable Sensors. leee Transactions on Information Technology in Biomedicine, 2009. 13(6): p. 864-873.

25. Salarian, A., et al., iTUG, a Sensitive and Reliable Measure of Mobility. leee Transactions on Neural Systems and Rehabilitation Engineering, 2010. 18(3): p. 303-310.

26. Zampieri, C., et al., The instrumented timed up and go test: potential outcome measure for disease modifying therapies in Parkinson's disease. Journal of Neurology Neurosurgery and Psychiatry, 2010. 81(2): p. 171-176.

27. Nilsson, F.M., et al., Digital movement analysis, a new objective method of measuring tardive dyskinesia and drug-induced parkinsonian tremor: acceptability, reliability and validity. Eur Arch Psychiatry Clin Neurosci, 1996. 246(2): p. 71-7.

28. Neelam, K., D. Garg, and M. Marshall, A systematic review and meta-analysis of neurological soft signs in relatives of people with schizophrenia. BMC Psychiatry, 2011. 11: p. 139.

29. Bachmann, S., et al., Neurological soft signs in the clinical course of schizophrenia: results of a metaanalysis. Front Psychiatry, 2014. 5: p. 185.

30. Morrens, M., W. Hulstijn, and B. Sabbe, Psychomotor slowing in schizophrenia. Schizophr Bull, 2007. 33(4): p. 1038-53.

31. Pappa, S. and P. Dazzan, Spontaneous movement disorders in antipsychotic-naive patients with firstepisode psychoses: a systematic review. Psychol Med, 2009. 39(7): p. 1065-76.

32. Howes, O.D., et al., Mechanisms underlying psychosis and antipsychotic treatment response in schizophrenia: insights from PET and SPECT imaging. Curr Pharm Des, 2009. 15(22): p. 2550-9.

33. Kar, N., S. Barreto, and R. Chandavarkar, Clozapine Monitoring in Clinical Practice: Beyond the Mandatory Requirement. Clin Psychopharmacol Neurosci, 2016. 14(4): p. 323-329.

34. Koning, J.P., et al., Movement disorders in nonpsychotic siblings of patients with nonaffective psychosis. Psychiatry Res, 2011. 188(1): p. 133-7.

35. Mittal, V.A., et al., Striatal volumes and dyskinetic movements in youth at high-risk for psychosis. Schizophr Res, 2010. 123(1): p. 68-70.

36. Mentzel, T.Q., et al., High Incidence and Prevalence of Drug-Related Movement Disorders in Young Patients With Psychotic Disorders. J Clin Psychopharmacol, 2017. 37(2): p. 231-238.

37. van Harten, P.N., et al., Movement disorders and psychosis, a complex marriage. Front Psychiatry, 2014. 5: p. 190.

38. Mittal, V.A. and E.F. Walker, Movement abnormalities predict conversion to Axis I psychosis among prodromal adolescents. J Abnorm Psychol, 2007. 116(4): p. 796-803.

39. Mittal, V.A., et al., Movement abnormalities and the progression of prodromal symptomatology in adolescents at risk for psychotic disorders. J Abnorm Psychol, 2007. 116(2): p. 260-7.

40. Mittal, V.A., et al., Longitudinal progression of movement abnormalities in relation to psychotic symptoms in adolescents at high risk of schizophrenia. Archives of general psychiatry, 2008. 65(2): p. 165-71.

41. Cortese, L., et al., Relationship of neuromotor disturbances to psychosis symptoms in first-episode neuroleptic-naive schizophrenia patients. Schizophr Res, 2005. 75(1): p. 65-75.

42. van Harten, P.N. and D.E. Tenback, Movement disorders should be a criterion for schizophrenia in DSM-V. Psychological medicine, 2009. 39(10): p. 1754-5; author reply 1755-6.

43. Raiszadeh, M.M., et al., Proteomic analysis of eccrine sweat: implications for the discovery of schizophrenia biomarker proteins. J Proteome Res, 2012. 11(4): p. 2127-39. 

